four digits of the final result. The result has been tabulated to $3089 \mathrm{D}$; the final digit is unrounded.

Running time for the $3093 \mathrm{D}$ was approximately thirteen minutes. The programming takes account of the number of zeros generated to the right of the decimal point in each factor, so that the number of operations required for each term in the series decreases. This leads to the following statement-if the time to compute $\pi$ to $m$ digits is $t$ units, then the time to produce $k m$ digits is roughly $k^{2} t$ units; this holds true as long as the calculation is contained in high-speed storage.

The following table gives a count of each of the digits in $\pi$.

(1)

$\begin{array}{cc} & 1-3090 \\ 0 & 269 \\ 1 & 315 \\ 2 & 314 \\ 3 & 276 \\ 4 & 322 \\ 5 & 326 \\ 6 & 311 \\ 7 & 297 \\ 8 & 318 \\ 9 & 342 \\ \Sigma & 3090\end{array}$

(2)

3090

315

314

76

326

11

18

42

090
(3)

1-2036

184

213

210

191

198

211

204

200

207

218

2036
(4)

2037-3090

85

102

104

85

124

115

107

97

111

124

1054
.46

.47

.50

.45

.63

.54

.52

.49

.54

.57

.52

S. C. Nicholson

J. JEENEL

Watson Scientific Computing Laboratory

612 West 115th Street

New York 27, New York

1. The IBM-Naval Ordnance Research Calculator, now located at Naval Proving Ground, Dahlgren, Virginia.

2. GEORGE W. REITwIESNER, "An ENIAC determination of $\pi$ and $e$ to more than 2000 decimal places," $M T A C$, v. 4,1950 , p. 11-15.

3. For a description of the NORC checking system, see W. J. Eckert \& R. B. Jones, Faster, Faster, McGraw-Hill Book Company, New York, 1955, p. 98-104.

\title{
Orthogonal Polynomials Arising in the Numerical Evaluation of Inverse Laplace Transforms
}

Abstract. In finding $f(t)$, the inverse LAPLACE transform of $F(p)$, where (1) $f(t)=(1 / 2 \pi j) \int_{c-j \infty}^{c+j \infty} e^{p t} F(p) d p$, the function $F(p)$ may be either known only numerically or too complicated for evaluating $f(t)$ by CAUCHY's theorem. When $F(p)$ behaves like a polynomial without a constant term, in the variable $1 / p$, along $(c-j \infty, c+j \infty)$, one may find $f(t)$ numerically using new quadrature formulas (analogous to those employing the zeros of the LAGUERRE polynomials in the direct Laplace transform). Suitable choice of $p_{i}$ yields an $n$-point quadrature 
formula that is exact when $\rho_{2 n}$ is any arbitrary polynomial of the (2n)th degree in $x \equiv 1 / p$ without a constant term, namely: (2) $(1 / 2 \pi j) \int_{c-j \infty}^{c+j \infty} e^{p} \rho_{2 n}(1 / p) d p$ $=\sum_{i=1}^{n} A_{i}{ }^{(n)} \rho_{2 n}\left(1 / p_{i}\right)$. In (2), $x_{i} \equiv 1 / p_{i}$ are the zeros of the orthogonal polynomials $p_{n}(x) \equiv \prod_{i=1}^{n}\left(x-x_{i}\right)$ where $(3)(1 / 2 \pi j) \int_{c-j \infty}^{c+j \infty} e^{p}(1 / p) p_{n}(1 / p)(1 / p)^{i} d p=0, i=0$, $1, \cdots, n-1$ and $A_{i}{ }^{(n)}$ correspond to the Christoffel numbers. The normalization $P_{n}(1 / p) \equiv(4 n-2)(4 n-6) \cdots 6 p_{n}(1 / p), n \geq 2$, produces all integral coefficients. $P_{n}(1 / p)$ is proven to be $(-1)^{n} e^{-p} p^{n} d^{n}\left(e^{p} / p^{n}\right) / d p^{n}$. The normalization factor is proved, in three different ways, to be given by (4) $(1 / 2 \pi j) \int_{c-j \infty}^{c+j \infty} e^{p}(1 / p)\left[P_{n}(1 / p)\right]^{2} d p=\frac{1}{2}(-1)^{n}$. Proofs are given for the recurrence formula (5) $(2 n-3) P_{n}(x)=[(4 n-2)(2 n-3) x+2] P_{n-1}(x)+(2 n-1) P_{n-2}(x)$, for $n \geq 3$, and the differential equation (6) $x^{2} P_{n}^{\prime \prime}(x)+(x-1) P_{n}^{\prime}(x)-n^{2} P_{n}(x)=0$. The quantities $p_{i}{ }^{(n)}, 1 / p_{i}{ }^{(n)}$ and $A_{i}{ }^{(n)}$ were computed, mostly to $6 \mathrm{~S}-8 \mathrm{~S}$, for $i=1(1) n, n=1(1) 8$.

I. Introduction : Occurrence of inverse Laplace transforms. For a given function of $p, F(p)$, which is the direct Laplace transform of some unknown function $f(t)$, for $t>0$, one usually finds the $f(t)$ from the following explicit expression:

$$
f(t)=\frac{1}{2 \pi j} \int_{c-j \infty}^{c+j \infty} e^{p t} F(p) d p .
$$

Formula (1) is known as the inverse Laplace transform of $F(p)$. In (1) the quantity $c$ is a real constant \pm 0 that is greater than the real part of all the singular points of $F(p)$. In practice $c$ is usually positive, but $c$ can be negative as long as for $f(t)$ satisfying Dirichlet's conditions in any finite positive interval the integral $\int_{0}^{\infty} e^{-c t} f(t) d t$ is absolutely convergent (H. S. CARsLAw and J. C. JAEger [1]).

A note by the referee follows this paper and indicates relations between the present work and work published elsewhere.

The examples treated in most textbooks on operational calculus and Laplace transforms contain such functions $F(p)$ that their poles and branch points (and residues also) are obtainable without too much difficulty, and the inversion integral in (1) is evaluated by suitable deformation of the path of integration, and the use of Cauchy's theorem. But there are countless other examples where $F(p)$ might be too complicated to yield explicit information about the location and nature of its singularities without a prohibitive amount of labor. For instance, one will recall that in most textbook examples treating the solution of ordinary and partial differential equations by operational means, the original system of differential equations is transformed into a system whose solution $F(p)$ is usually some known elementary function or a very extensively tabulated function of a simple differential equation (like a Bessel function), so that its analytic character and singularities are well known. But in actual practice one might not be fortunate enough to obtain such a comparatively simple $F(p)$. Thus the transformed differential equations might not yield a known function. Instead it might be amenable 
only to solution in series or numerical integration, with an $F(p)$ that is given as a tabulated function of $p$. Also, the solution $F(p)$ might be given explicitly in closed form as a combination of integrals of such complicated analytic expressions that it might be easier to evaluate it for different numerical values of $p$ than to find its poles, residues, and branch points.

The purpose of this present article is to discuss the properties of a new set of orthogonal polynomials which can be the basis for convenient formulas for approximating $f(t)$ in (1) for different positive values of $t$ when one has an $F(p)$ that is too complicated to show its analytic character, but which can be calculated for any $p$.

All further discussion will now be for $F(p)$ assumed to be exactly of the form $\sum_{r=1}^{m} \frac{a_{r}}{p^{r}}$, i.e., a polynomial in $1 / p$ without a constant term.

To obtain a definite integral without a parameter $t$ in the exponential term, which is the "weight function," let $p t=u$ in (1), so that we obtain

$$
f(t)=\frac{1}{2 \pi j t} \int_{c_{1}-j \infty}^{c_{1}+j \infty} e^{u} F\left(\frac{u}{t}\right) d u,
$$

where $F\left(\frac{u}{t}\right)$ is still a polynomial in $1 / u$, without a constant term.

II. Use of orthogonal polynomials. At this point one may recall the application of the theory of orthogonal polynomials to quadrature formulas for definite integrals where the integrand is the product of a preassigned weight function and a polynomial $P(t)$. There it is possible to employ the value of $P(t)$ at $n$ fixed irregularly spaced points $t_{i}, i=1,2, \cdots, n$, such that the resulting quadrature formula is exact when $P(t)$ is any arbitrary polynomial of $(2 n-1)$-th degree. Thus for the direct Laplace transform of $P(t)$, namely $\int_{0}^{\infty} e^{-p t} P(t) d t$, which is essentially $\int_{0}^{\infty} e^{-t} Q(t) d t$ for polynomial $Q(t)$, the points $t_{i}$ are taken equal to the zeros of the Laguerre polynomials, which have been tabulated extensively (H. E. SAlZER and R. ZuCker [2]). In the present case, even though we are not dealing with a polynomial in $p$, we can still solve the problem of finding a Gaussian-type quadrature formula for $\left(1^{\prime}\right)$ of approximately double the degree of accuracy of an ordinary quadrature formula based upon the same number of equally spaced points.

Thus let $\rho_{2 n}(1 / p)$ be any arbitrary $(2 n)$-th degree polynomial in the variable $1 / p$, which vanishes at $1 / p=0$. Consider $n$ distinct points $1 / p_{i}, i=1,2, \cdots, n$ other than $1 / p=0$ and construct the $(n+1)$-point Lagrangian polynomial approximation (of the $n^{\text {th }}$ degree in $1 / p$ ), to $\rho_{2 n}(1 / p)$, based upon the points $1 / p_{i}$, $i=1,2, \cdots, n$ and $1 / p=0$. The $(n+1)$ th point $1 / p=0$ is needed in order to provide for the property that $\rho_{2 n}(1 / p)$ vanishes at $p=\infty$. We have for this polynomial approximation $L^{(n+1)}(1 / p)$ the explicit expression

$$
L^{(n+1)}\left(\frac{1}{p}\right)=\sum_{i=1}^{n+1} L_{i}^{(n+1)}\left(\frac{1}{p}\right) \rho_{2 n}\left(\frac{1}{p_{i}}\right),
$$


where on the right hand side of (2)

$$
L_{i}^{(n+1)}\left(\frac{1}{p}\right) \equiv \prod_{k=1}^{n+1}\left(\frac{1}{p}-\frac{1}{p_{k}}\right) / \prod_{k=1}^{n+1}\left(\frac{1}{p_{i}}-\frac{1}{p_{k}}\right),
$$

the $\Pi^{\prime}$ denoting the absence of $k=i$. In (2), $p_{n+1}$ is $\infty$, so that there is actually no $(n+1)$-th term in (2) and $L_{n+1}^{(n+1)}(1 / p)$ is not used. (The summation in (2) is written with $n+1$ instead of $n$ to avoid confusion with the $(n-1)$-th degree coefficients $L_{i}^{(n)}(1 / p)$ which differ from the $L_{i}^{(n+1)}(1 / p)$ by not having the factor $p_{i} / p$.)

Following the method in G. SzEGö [3], we consider the $(2 n)$-th degree polynomial in $1 / p$, namely, $\rho_{2 n}(1 / p)-L^{(n+1)}(1 / p)$ which vanishes at $1 / p=0,1 / p_{i}$, $i=1,2, \cdots, n$, and thus has

as a factor. Writing

$$
\frac{1}{p} p_{n}\left(\frac{1}{p}\right) \equiv \frac{1}{p} \prod_{i=1}^{n}\left(\frac{1}{p}-\frac{1}{p_{i}}\right)
$$

$$
\rho_{2 n}\left(\frac{1}{p}\right)=L^{(n+1)}\left(\frac{1}{p}\right)+\frac{1}{p} p_{n}\left(\frac{1}{p}\right) r_{n-1}\left(\frac{1}{p}\right),
$$

it follows that

$$
\text { (5) } \begin{aligned}
\frac{1}{2 \pi j} \int_{c-j \infty}^{c+j \infty} e^{p} \rho_{2 n}\left(\frac{1}{p}\right) d p=\frac{1}{2 \pi j} \int_{c-j \infty}^{c+j \infty} e^{p} L^{(n+1)}\left(\frac{1}{p}\right) d p \\
+\frac{1}{2 \pi j} \int_{c-j \infty}^{c+j \infty} e^{p} \frac{1}{p} p_{n}\left(\frac{1}{p}\right) r_{n-1}\left(\frac{1}{p}\right) d p .
\end{aligned}
$$

Thus if the second term in the right member of (5) always vanishes, (5) will be an $n$-point quadrature formula that is exact for any $(2 n)$-th degree polynomial in $1 / p$ without a constant term, namely,

$$
\frac{1}{2 \pi j} \int_{c-j \infty}^{c+j \infty} e^{p} \rho_{2 n}\left(\frac{1}{p}\right) d p=\sum_{i=1}^{n} A_{i}^{(n)} \rho_{2 n}\left(\frac{1}{p_{i}}\right),
$$

where the "Christoffel numbers" $A_{i}{ }^{(n)}$ are given by [6]

$$
A_{i}^{(n)} \equiv \frac{1}{2 \pi j} \int_{c-j \infty}^{c+j \infty} e^{p} L_{i}^{(n+1)}\left(\frac{1}{p}\right) d p
$$

A sufficient condition for (6) to hold is obviously the "orthogonality" of $(1 / p) p_{n}(1 / p)$ with respect to any arbitrary $\rho_{n-1}(1 / p)$, namely,

$$
\frac{1}{2 \pi j} \int_{c-j \infty}^{c+j \infty} e^{p} \frac{1}{p} p_{n}\left(\frac{1}{p}\right)\left(\frac{1}{p}\right)^{i} d p=0, \quad i=0,1, \cdots, n-1 .
$$

The necessity of (8) is also obvious from (6) by choosing

$$
\rho_{2 n}(1 / p)=(1 / p) p_{n}(1 / p) \rho_{n-1}(1 / p)
$$

where $\rho_{n-1}(1 / p)$ is any arbitrary polynomial in $1 / p$ of the $(n-1)$-th degree. 
Hence the points $1 / p_{i}$, now denoted by $1 / p_{i}{ }^{(n)}$, are the zeros of a certain set of orthogonal polynomials in the variable $1 / p$.

The condition of orthogonality (8) is also mathematically equivalent, in terms of actual polynomials (by setting $x=1 / p$ ), to having a polynomial of the $n^{\text {th }}$ degree $q_{n}(x)$ which is orthogonal to any $\rho_{n-1}(x)$, with weight function $e^{1 / x} / x$, where the path of integration is a circle of radius $1 / 2 c$ whose center is at $(1 / 2 c, 0)$.

If the polynomial $p_{n}(1 / p)$ is written as

$$
\left(\frac{1}{p}\right)^{n}+b_{n-1}\left(\frac{1}{p}\right)^{n-1}+b_{n-2}\left(\frac{1}{p}\right)^{n-2}+\cdots+b_{1}\left(\frac{1}{p}\right)+b_{0}
$$

the determination of $b_{i}, i=0,1, \cdots, n-1$, to satisfy the conditions of orthogonality (8), making use of

$$
\frac{1}{2 \pi j} \int_{c-j \infty}^{c+j \infty} \frac{e^{p}}{p^{m+1}} d p=\frac{1}{m !}
$$

is in the solution of this system of linear equations:

$$
\left\{\begin{array}{l}
\frac{1}{n !}+\frac{b_{n-1}}{(n-1) !}+\frac{b_{n-2}}{(n-2) !}+\cdots+\frac{b_{1}}{1 !}+\frac{b_{0}}{0 !}=0 \\
\frac{1}{(n+1) !}+\frac{b_{n-1}}{n !}+\frac{b_{n-2}}{(n-1) !}+\cdots+\frac{b_{1}}{2 !}+\frac{b_{0}}{1 !}=0 \\
\cdot \cdot \cdot \cdot \cdot \cdot \cdot \cdot \cdot \cdot \cdot \cdot \cdot \cdot \cdot \cdot+\frac{b_{1}}{n !}+\frac{b_{0}}{(n-1) !}=0 . \\
\frac{1}{(2 n-1) !}+\frac{b_{n-1}}{(2 n-2) !}+\frac{b_{n-2}}{(2 n-3) !}+\cdots+
\end{array}\right.
$$

For numerical work it is somewhat easier to solve $\left(9^{\prime}\right)$ in the form

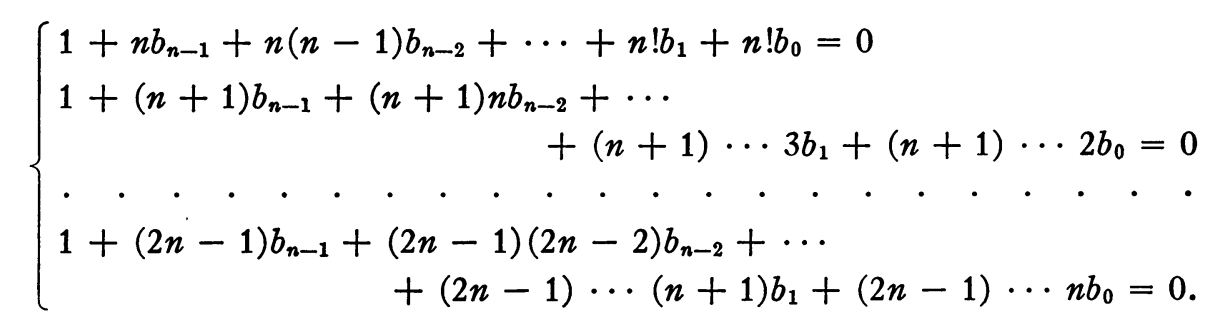

III. Explicit expression for orthogonal polynomials. It is convenient to normalize the polynomials $p_{n}(x)$, where $x \equiv 1 / p$, by multiplying $p_{n}(x)$, for $n \geq 2$, by $(4 n-2)(4 n-6) \cdots 6$. This normalization produces polynomials with all coefficients integral (proven below) and it is not the usual normalization by multiplication by

$$
\left[\frac{1}{2 \pi j} \int_{c-j \infty}^{c+j \infty} e^{p} \frac{1}{p}\left\{p_{n}\left(\frac{1}{p}\right)\right\}^{2} d p\right]^{-\frac{1}{2}} .
$$

Denoting $(4 n-2)(4 n-6) \cdots 6 p_{n}(1 / p)$ by $P_{n}(1 / p)$ for $n \geq 2$, and $p_{1}(1 / p)$ by $P_{1}(1 / p)$, one can avoid the labor of solving $\left(9^{\prime}\right)$ or $(9)$ directly by showing that 
$P_{n}(1 / p)$ has the following more elegant definition:

$$
P_{n}\left(\frac{1}{p}\right)=(-1)^{n} e^{-p} p^{n} \frac{d^{n}}{d p^{n}}\left(\frac{e^{p}}{p^{n}}\right)
$$

That (10) yields the leading coefficient of $1 / p^{n}$ in $P_{n}(1 / p)$, namely

$$
\left\{\begin{array}{l}
1, \quad \text { for } n=1, \\
(4 n-2)(4 n-6) \cdots 6, \text { for } n \geq 2,
\end{array}\right.
$$

is obvious by induction. To prove the orthogonality property, or (8), it suffices to prove the vanishing of

$$
\frac{1}{2 \pi j} \int_{c-j \infty}^{c+j \infty} e^{p} \frac{1}{p}\left[(-1)^{n} e^{-p} p^{n} \frac{d^{n}}{d p^{n}}\left(\frac{e^{p}}{p^{n}}\right)\right]\left[(-1)^{m} e^{-p} p^{m} \frac{d^{m}}{d p^{m}}\left(\frac{e^{p}}{p^{m}}\right)\right] d p
$$

for $m<n$. This last expression is written as

$$
\frac{(-1)^{m+n}}{2 \pi j} \int_{c-j \infty}^{c+j \infty} e^{-p} p^{m+n-1} \frac{d^{n}}{d p^{n}}\left(\frac{e^{p}}{p^{n}}\right) \frac{d^{m}}{d p^{m}}\left(\frac{e^{p}}{p^{m}}\right) d p,
$$

and after integrating by parts $m$ times, noting that the integrated parts always vanish, we have

$$
\frac{(-1)^{m}(-1)^{m+n}}{2 \pi j} \int_{c-j \infty}^{c+j \infty} \frac{d^{m}}{d p^{m}}\left[e^{-p} p^{m+n-1} \frac{d^{n}}{d p^{n}}\left(\frac{e^{p}}{p^{n}}\right)\right] \frac{e^{p}}{p^{m}} d p,
$$

which by LeIBNITz's rule is expressible as

(D) $\quad \frac{(-1)^{m}(-1)^{m+n}}{2 \pi j} \int_{c-j \infty}^{c+j \infty} \sum_{r=0}^{m}\left(\begin{array}{c}m \\ r\end{array}\right) \frac{d^{r}}{d p^{r}}\left(e^{-p} p^{m+n-1}\right) \frac{d^{m-r+n}}{d p^{m-r+n}}\left(\frac{e^{p}}{p^{n}}\right) \frac{e^{p}}{p^{m}} d p$.

Application of Leibnitz's rule a second time to

$$
\frac{d^{r}}{d p^{r}}\left(e^{-p} p^{m+n-1}\right)
$$

in the above and cancellation of $e^{p} / p^{m}$, yields

$$
\text { (E) } \begin{array}{r}
\frac{(-1)^{m}(-1)^{m+n}}{2 \pi j} \int_{c-j \infty}^{c+j \infty} \sum_{r=0}^{m}\left(\begin{array}{l}
m \\
r
\end{array}\right)\left[\sum_{s=0}^{r}(-1)^{r-s}\left(\begin{array}{l}
r \\
s
\end{array}\right)\left(\begin{array}{c}
m+n-1 \\
s
\end{array}\right) s ! p^{n-1-s}\right] \\
\times \frac{d^{m-r+n}}{d p^{m-r+n}}\left(\frac{e^{p}}{p^{n}}\right) d p .
\end{array}
$$

Now we integrate by parts $(m-r+n)$ times each term of the above double summation. The integrated part will always vanish since it will have a factor of $1 / p$ to at least the first power. Furthermore, at some stage in the partial integration of each term, that stage varying with the term, the integral part will also vanish if $m<n$. This last follows because the lowest power of $p^{n-1-s}$ is positive or zero, since $s$ can equal at the most $r$ which can equal at the most $m \leq n-1$. Then in the integration by parts the positive or zero power $p^{n-1-s}$, for each value 
of $s$ between 0 and $r$, would always be eventually annulled because the initially occurring differential operator $d^{m-r+n} / d p^{m-r+n}$ is of order $m-r+n>n-1-s$ even for the highest value of $n-1-s$ when $s=0$ (due to $m-r+n>n-1$ for every $r$ between 0 and $m$ ). Thus (E) vanishes, which proves (10), and establishes at the same time that this normalization yields all integral coefficients for $P_{n}(1 / p)$.

IV. Normalization factor. To obtain the normalization factor, which turns out to be given by

$$
\frac{1}{2 \pi j} \int_{c-j \infty}^{c+j \infty} e^{p} \frac{1}{p}\left[P_{n}\left(\frac{1}{p}\right)\right]^{2} d p=\frac{1}{2}(-1)^{n},
$$

we repeat the preceding argument for $m=n$ and now notice that in the final integral (E) the lowest power of $p^{n-1-s}$ will survive the integration by parts, because it is equal to $1 / p$. Retaining in the double summation in $(E)$ only the single non-vanishing term $s=r=m=n$, we get

$$
\frac{(-1)^{2 n+n}}{2 \pi j} \int_{c-j \infty}^{c+j \infty}\left(\begin{array}{c}
2 n-1 \\
n
\end{array}\right) n ! \frac{1}{p} \frac{d^{n}}{d p^{n}}\left(\frac{e^{p}}{p^{n}}\right) d p
$$

which is integrated by parts $n$ times, the integrated part always vanishing, to give

$$
\frac{(-1)^{2 n+2 n}}{2 \pi j}\left(\begin{array}{c}
2 n-1 \\
n
\end{array}\right) n ! \int_{c-j \infty}^{c+j \infty} e^{p} \frac{(-1)^{n} \cdot 2 \cdot 3 \cdots(n-1) n}{p^{2 n+1}} d p .
$$

But $(G)$ is

$$
(-1)^{n}\left(\begin{array}{c}
2 n-1 \\
n
\end{array}\right) n ! n ! \frac{1}{(2 n) !}=\frac{(-1)^{n}(2 n-1)(2 n-2) \cdots n}{n !} n ! n ! \frac{1}{(2 n) !}
$$

which reduces to $(-1)^{n} n / 2 n$ or $\frac{1}{2}(-1)^{n}$, thus proving (11).

From (10), the explicit formula for $P_{n}(1 / p)$ is seen to be [7]

$$
\begin{aligned}
P_{n}\left(\frac{1}{p}\right)= & (-1)^{n}\left[\frac{(-1)^{n}\left(\begin{array}{c}
2 n-1 \\
n
\end{array}\right) n !}{p^{n}}\right. \\
& +\frac{(-1)^{n-1}\left(\begin{array}{l}
n \\
1
\end{array}\right)\left(\begin{array}{c}
2 n-2 \\
n-1
\end{array}\right)(n-1) !}{p^{n-1}} \\
& +\frac{(-1)^{n-2}\left(\begin{array}{l}
n \\
2
\end{array}\right)\left(\begin{array}{c}
2 n-3 \\
n-2
\end{array}\right)(n-2) !}{p^{n-2}}+\cdots \\
+ & \left.\frac{(-1)^{n-r}\left(\begin{array}{l}
n \\
r
\end{array}\right)\left(\begin{array}{c}
2 n-r-1 \\
n-r
\end{array}\right)(n-r) !}{p^{n-r}}+\frac{n^{2}(-1)^{1}}{p}+(-1)^{0}\right]
\end{aligned}
$$


V. Recurrence formula. It is easy to obtain the recurrence relation for the polynomials $P_{n}(x)$ by employing a fundamental theorem about the existence of a recurrence formula connecting any three successive orthogonal polynomials (G. Szegö [8]), namely,

$$
P_{n}(x)=\left(a_{n} x+b_{n}\right) P_{n-1}(x)+c_{n} P_{n-2}(x) .
$$

Thus $a_{n}$ is immediately seen to be $4 n-2$. Equating constant terms in (13), one finds $c_{n}=b_{n}+1$, and after substitution into the equation derived from the coefficients of $x$, one obtains

$$
b_{n}=\frac{2}{2 n-3}, \quad c_{n}=\frac{2 n-1}{2 n-3},
$$

so that the recurrence formula satisfied by $P_{n}(x)$ is seen to be [9]

$$
\begin{array}{r}
(2 n-3) P_{n}(x)=[(4 n-2)(2 n-3) x+2] P_{n-1}(x)+(2 n-1) P_{n-2}(x) \\
\text { for } n \geq 3 .
\end{array}
$$

From (14) and (8) only, without making use of (10), one can again find the normalization factor given in (11), through the following inductive argument:

Multiply (14) by $P_{n-2}(x)$ and then operate with

$$
\frac{1}{2 \pi j} \int_{c-j \infty}^{c+j \infty} e^{p} \frac{1}{p} \cdots d p
$$

to obtain (making use of $(8))$ :

$$
\begin{aligned}
0=\frac{(4 n-2)(2 n-3)}{2 \pi j} \int_{c-j \infty}^{c+j \infty} e^{p} \frac{1}{p} P_{n-1}\left(\frac{1}{p}\right) \cdot \frac{1}{p} P_{n-2}\left(\frac{1}{p}\right) d p & \\
& +0+\frac{(2 n-1)}{2 \pi j} \int_{c-j \infty}^{c+j \infty} e^{p} \frac{1}{p}\left[P_{n-2}\left(\frac{1}{p}\right)\right]^{2} d p .
\end{aligned}
$$

Denoting the left member of (11) by $F_{n}$, still making use of (8) to replace in the first of the above integrals $(1 / p) P_{n-2}(1 / p)$ by

$$
\frac{1}{a_{n-1}} P_{n-1}\left(\frac{1}{p}\right)=\frac{1}{4 n-6} P_{n-1}\left(\frac{1}{p}\right),
$$

one now obtains

$$
0=\frac{(4 n-2)(2 n-3)}{4 n-6} F_{n-1}+(2 n-1) F_{n-2},
$$

or $F_{n-1}=-F_{n-2}$. Since $F_{1}=-\frac{1}{2}$, (11) follows by induction.

The normalization given in (11) can be seen in a third way, directly from the 
explicit formula for $P_{n}(1 / p)$ in (12). For, in view of (8), it suffices to consider only

$$
\frac{1}{2 \pi j} \int_{c-j \infty}^{c+j \infty} \frac{e^{p}}{p} \frac{(-1)^{2 n}}{p^{n}}\left(\begin{array}{c}
2 n-1 \\
n
\end{array}\right) n ! P_{n}\left(\frac{1}{p}\right) d p
$$

or

$$
(-1)^{2 n}\left(\begin{array}{c}
2 n-1 \\
n
\end{array}\right) n ! \sum_{r=0}^{n} \frac{(-1)^{2 n-r}\left(\begin{array}{l}
n \\
r
\end{array}\right)\left(\begin{array}{c}
2 n-r-1 \\
n-r
\end{array}\right)(n-r) !}{(2 n-r) !}
$$

or

(J) $\left(\begin{array}{c}2 n-1 \\ n\end{array}\right) n ! \sum_{r=0}^{n}(-1) r \frac{n(n-1) \cdots(n-r+1)}{r !} \frac{(2 n-r-1) \cdots(n+1) n}{(2 n-r)(2 n-r-1) \cdots(n+1) n !}$,

which, after cancellations, is written as

$$
\sum_{r=0}^{n}(-1)^{r} \frac{(2 n-1)(2 n-2) \cdots n}{(n-r) ! n !} \frac{n}{2 n-r} \frac{n(n-1) \cdots(n-r+1)(n-r) !}{r !},
$$

or

$$
n \sum_{r=0}^{n}(-1)^{r} \frac{1}{2 n-r} \frac{(2 n-1)(2 n-2) \cdots n}{(n-r) ! r !},
$$

and this, in turn, is expressible in the form

$$
\begin{aligned}
& \frac{1}{2}(-1)^{n} \\
& \quad \times \sum_{r=0}^{n} \frac{(2 n-0)(2 n-1)(2 n-2) \cdots(2 n-[r-1])(2 n-[r+1]) \cdots(2 n-n)}{(r-0)(r-1) \cdots(r-[r-1])(r-[r+1]) \cdots(r-n)} .
\end{aligned}
$$

In $(M)$, the $\frac{1}{2}(-1)^{n}$ is multiplied by the sum of the coefficients of the Lagrangian interpolation polynomial for the $(n+1)$ points $0,1, \cdots, n$, for the variable equal to $2 n$. But that sum is identically equal to 1 , i.e., for any value, $2 n$ or otherwise. Thus we obtain once more $\frac{1}{2}(-1)^{n}$ for the normalization.

VI. Integral coefficients. It may be of interest to show that (14) alone, without any knowledge of (10), implies that $P_{n}(x)$ has integral coefficients. We prove this by noting that $P_{n+1}(x)$ will have integral coefficients if $P_{m}(x), m \leq n$, has integral coefficients and the following identical polynomial congruence holds for $m=n+1$ :

$$
2 P_{m-1}(x)+(2 m-1) P_{m-2}(x) \equiv 0 \quad(\bmod (2 m-3)) .
$$

Now the existence of integral coefficients of $P_{m}(x)$ and congruence (15) can be verified for the first few values of $m$. We then show that if (15) holds for some particular $m=n$, it holds for $m=n+1$, provided $P_{m}(x), m \leq n-1$, has 
integral coefficients, or, in other words, that

$$
2 P_{n}(x)+(2 n+1) P_{n-1}(x) \equiv 0 \quad(\bmod (2 n-1)) .
$$

This last congruence, by (14), is equivalent to

$\frac{4}{2 n-3} P_{n-1}(x)+\frac{2(2 n-1)}{2 n-3} P_{n-2}(x)+(2 n+1) P_{n-1}(x) \equiv 0 \quad(\bmod (2 n-1))$,

or to

$$
\frac{(2 n-1)^{2}}{2 n-3} P_{n-1}(x)+\frac{2(2 n-1)}{2 n-3} P_{n-2}(x) \equiv 0 \quad(\bmod (2 n-1))
$$

which in turn is expressible as

$$
(2 n-1)\left[\frac{(2 n-1) P_{n-1}(x)+2 P_{n-2}(x)}{2 n-3}\right] \equiv 0 \quad(\bmod (2 n-1)),
$$

or

$$
\begin{aligned}
&(2 n-1)\left[\frac{\left(2+(2 n-3) P_{n-1}(x)+(2 n-1-(2 n-3)) P_{n-2}(x)\right.}{2 n-3}\right] \equiv 0 \\
&(\bmod (2 n-1)) .
\end{aligned}
$$

But under the assumptions that (15) holds for $m=n$, and that $P_{m}(x), m \leq n-1$, has integral coefficients, the last quantity in brackets is a polynomial with integral coefficients, which shows that the last congruence is satisfied identically in $x$. Thus (15) holds for $m=n+1$ and $P_{n+1}(x)$ has integral coefficients. We proceed in this way to every $n$. There is a slight subtlety in the argument of this induction in the sense that the integral coefficients of $P_{m}(x)$ up to $m=n-1$ only are needed to go from $m=n$ to $m=n+1$ in (15), but then use is made of the integral coefficients of $P_{n}(x)$ in using (14) with $n+1$ in place of $n$.

VII. Differential equation. It is easy to show that $P_{n}(x)$ satisfies the differential equation

$$
x^{2} P_{n}^{\prime \prime}(x)+(x-1) P_{n}^{\prime}(x)-n^{2} P_{n}(x)=0
$$

Thus one merely expresses (12) in the form

$$
P_{n}(x)=(-1)^{n}\left[1+\sum_{r=1}^{n} \frac{(-1)^{r} n^{2}\left(n^{2}-1^{2}\right)\left(n^{2}-2^{2}\right) \cdots\left(n^{2}-\overline{r-1}\right)^{2} x^{r}}{r !}\right]
$$

and then observes that $\left(12^{\prime}\right)$ is equivalent to the automatically terminating "infinite series."

$$
P_{n}(x)=\sum_{r=0}^{\infty} a_{r} x^{r}
$$


where

$$
a_{0}=(-1)^{n} \text {, and } r a_{r}=-\left(n^{2}-\overline{r-1}^{2}\right) a_{r-1}, \text { for } r>0 \text {. }
$$

Working backwards from $\left(12^{\prime \prime}\right)$, by equating coefficients of $x^{r-1}$, one sees that $\left(12^{\prime \prime}\right)$ must arise from (16).

VIII. Explicit expressions for polynomials. Because these polynomials $P_{n}(x)$ are of fundamental importance, and their role in the inverse Laplace transform is comparable to the role of the Laguerre polynomials in the direct Laplace transform, their explicit expressions are given below for $n=1(1) 12$ :

$$
\begin{aligned}
& P_{1}(x)=x-1 \\
& P_{2}(x)=6 x^{2}-4 x+1 \\
& P_{3}(x)=60 x^{3}-36 x^{2}+9 x-1 \\
& P_{4}(x)=840 x^{4}-480 x^{3}+120 x^{2}-16 x+1 \\
& P_{5}(x)=15120 x^{5}-8400 x^{4}+2100 x^{3}-300 x^{2}+25 x-1 \\
& P_{6}(x)=332640 x^{6}-181440 x^{5}+45360 x^{4}-6720 x^{3}+630 x^{2}-36 x+1 \\
& P_{7}(x)=8648640 x^{7}-4656960 x^{6}+1164240 x^{5}-176400 x^{4}+17640 x^{3} \\
& -1176 x^{2}+49 x-1 \\
& P_{8}(x)=259459200 x^{8}-138378240 x^{7}+34594560 x^{6}-5322240 x^{5} \\
& +554400 x^{4}-40320 x^{3}+2016 x^{2}-64 x+1 \\
& P_{9}(x)=8821612800 x^{9}-4670265600 x^{8}+1167566400 x^{7}-181621440 x^{6} \\
& +19459440 x^{5}-1496880 x^{4}+83160 x^{3}-3240 x^{2}+81 x-1 \\
& P_{10}(x)=335221286400 x^{10}-176432256000 x^{9}+44108064000 x^{8}-69189 \\
& 12000 x^{7}+756756000 x^{6}-60540480 x^{5}+3603600 x^{4}-158400 x^{3} \\
& +4950 x^{2}-100 x+1 \\
& P_{11}(x)=14079294028800 x^{11}-7374868300800 x^{10}+1843717075200 x^{9} \\
& -291113222400 x^{8}+32345913600 x^{7}-2663781120 x^{6}+1664 \\
& 86320 x^{5}-7927920 x^{4}+283140 x^{3}-7260 x^{2}+121 x-1 \\
& P_{12}(x)=647647525324800 x^{12}-337903056691200 x^{11}+84475764172800 x^{10} \\
& -13408851456000 x^{9}+1508495788800 x^{8}-127031224320 x^{7} \\
& +8233505280 x^{6}-415134720 x^{5}+16216200 x^{4}-480480 x^{3} \\
& +10296 x^{2}-144 x+1 \text {. }
\end{aligned}
$$

IX. Zeros and Christoffel numbers. In the numerical table below there are given the values of the reciprocals of the zeros of $P_{n}(x)$ or $p_{i}{ }^{(n)}$, the zeros of $P_{n}(x)$, or $1 / p_{i}{ }^{(n)}$, and the corresponding Christoffel numbers $A_{i}{ }^{(n)}$, for $n=1(1) 8$. Use of these quantities in the quadrature formula (6) above can give theoretically exact accuracy for any polynomial in $1 / p$ (with no constant term) up to the 16 th degree. However, the fact that these tabulated values of $p_{i}{ }^{(n)}, 1 / p_{i}{ }^{(n)}$ and $A_{i}{ }^{(n)}$ are correct to only about a unit in the last significant figure that is given, must 


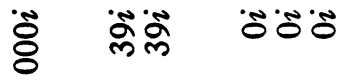

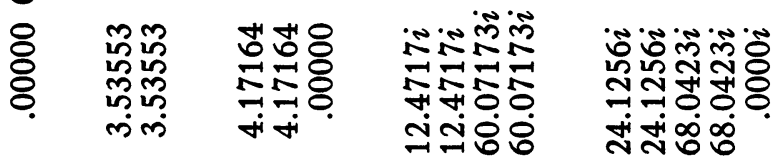

$$
\begin{aligned}
& \text { ह }+1++1+11+1++1+ \\
& 8 \quad 889 a \infty
\end{aligned}
$$

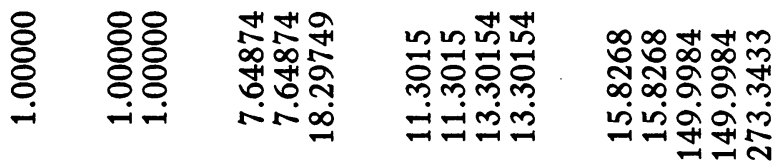

$$
\begin{aligned}
& \begin{array}{llllll}
2 & 1 & 1 & 1 & 1 & 1
\end{array}
\end{aligned}
$$

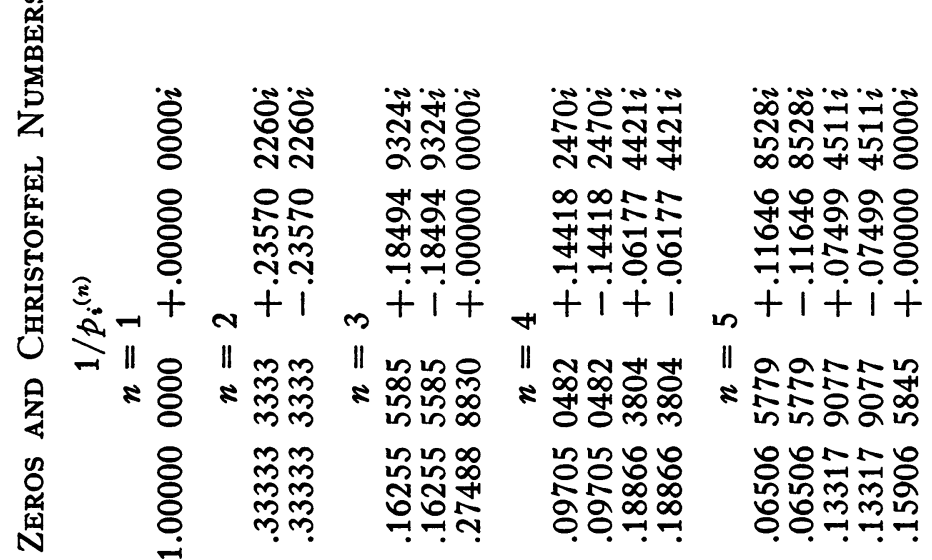

$$
\begin{aligned}
& \text { c }
\end{aligned}
$$

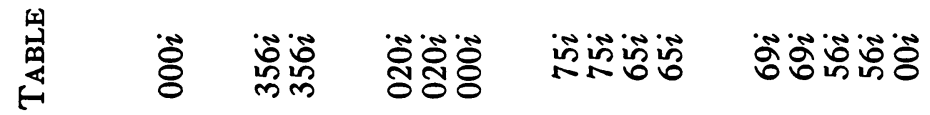

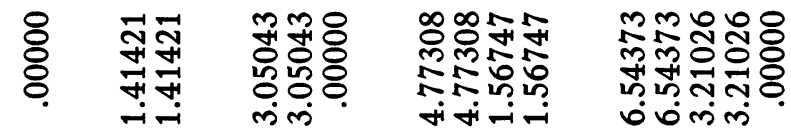

$$
\begin{aligned}
& \text { ह }+1+1++1+1+1+1+
\end{aligned}
$$

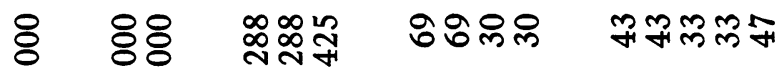

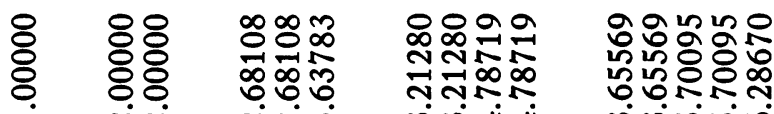

$$
\begin{aligned}
& \text { ㄱำ }
\end{aligned}
$$

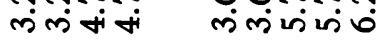

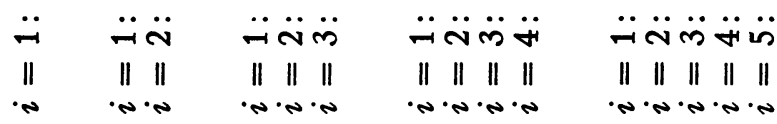




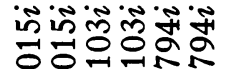 O० -7?

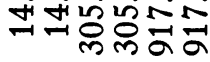 \\ ह. $1++11+$}

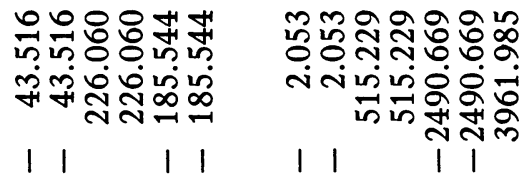

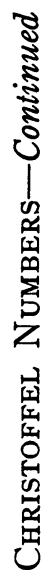

忩

:

点

cas

됩

\section{$0+i+i+1$ \\ - II N-inos

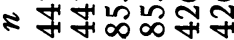 \\ ณที่กิก \\ ํㅟㅇํㅇ으소 \\ 0.000.7.}

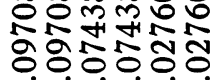

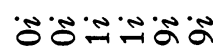

응죽구워

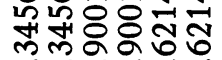
$\infty \infty+\dot{+}+\dot{-i}$

$\stackrel{Ð}{E}$

$1+1+1+$

$\infty \infty m m 0$

岁先的艺 m

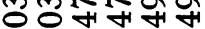
संपण

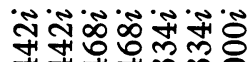

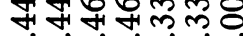

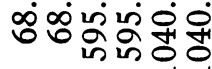

$+11++1+$

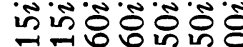

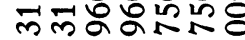

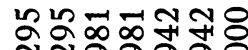

oㅇoㅇํㅇㅇㅇ

- $+i+i+i+$

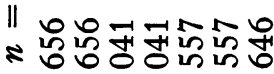

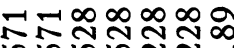
ชิำㅇํㅇํำ

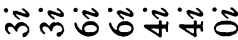

ㅇํㅇㅇㅇㅇㅎㄷㄷㅇㅇㅇ

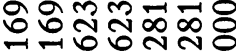
-70.

$1+1+1++$

thin m n

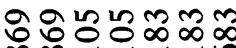

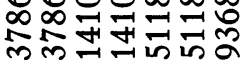
संत्

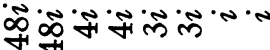

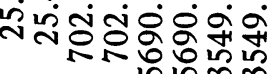

$1+1++17+$

ガずดดนn

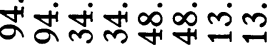
mo I

ํำ.4.

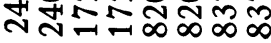

누궂ㅇㅇㅇㅛ สสษ

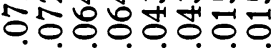
$\infty+1+i+i+1$

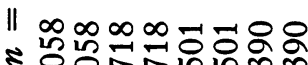

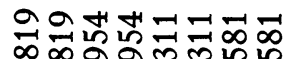

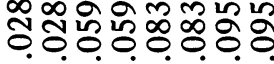

Dก

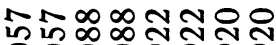
잉의 의

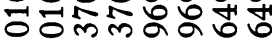

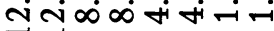
$1+1+1+1+$ $\ln \ln 000044$

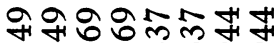
ผ $\infty \infty m \infty$ +irira åo $\ddot{\sim} \ddot{\sim} \ddot{\sim} \ddot{\gamma} \ddot{\sim} \ddot{\sigma}$

|| || || || || ||

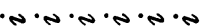

$\ddot{\sim} \ddot{\sim} \ddot{m} \ddot{\gamma} \ddot{\sim} \ddot{\sigma}$

|| || || || || || ||
|| || || || || || || || 
be taken into account in any practical example where some upper bound for the error should be estimated.

Ordnance Corps

Herbert E. SAlzer

Diamond Ordnance Fuze Laboratories

Washington, D. C.

NOTE BY REFEREE

The function $F(p)$ is subject to certain restrictions because it is a Laplace transform. In order for $F(p)$ to be the Laplace transform of the function $f(t)$ given by (1), it is sufficient that $F(p)$ have the form (cf. G. DOETSCH [5]):

$$
F(p)=a / p+F_{1}(p) / p^{1+\delta}
$$

where $\delta>0, a$ is a constant, and $F_{1}(p)$ is analytic and bounded in the half plane $\operatorname{Re}(p)>c$. We assume that this condition is satisfied. Whether this condition is also sufficient for the convergence of the $n$-point quadrature formula to the true value of $f(t)$ in (1), when $n$ tends to infinity, has not been determined. The author makes use here of the fact that the convergence occurs whenever $F(p)$ is a polynomial in $1 / p$ without a constant term; in fact, the quadrature is exact for polynomials of degree not greater than $2 n$. G. SzEGö $[10]$ has shown that under quite general conditions a Gauss-Jacobi type quadrature formula which converges for polynomials also converges for a much wider class of functions. Unfortunately his theorems do not seem to apply directly to the present case because the integral (1) involves a complex valued weight function which is not of bounded variation.

1. H. S. Carslaw \& J. C. Jaeger, Operational Methods in Applied Mathematics, 2nd edition, Oxford University Press, 1949, p. 75.

2. H. E. SALZER \& R. ZUCKER, "Table of the Zeros and Weight Factors of the First Fifteen Laguerre Polynomials," Amer. Math. Soc., Bull., v. 55, 1949, p. 1004-1012.

3. G. SzEgö, Orthogonal Polynomials, Amer. Math. Soc., Colloquium Pub., v. 23, 1939, p. 46-47.

4. H. L. KRaLl \& O. Frink, "A New Class of Orthogonal Polynomials: The Bessel Polynomials," Amer. Math. Soc., Trans., v. 65, 1, 1949, p. 100-115.

5. G. DoETsch, Theorie und Anwendung der Laplace-Transformation, Springer, Berlin, 1937, p. 128.

6. The shift in notation from $(n+1)$ to $n$ in $A_{i}{ }^{(n)}$ will cause no confusion after the $A_{i}$ 's have been computed and are ready for use in (6).

7. It was called to the author's attention by. H. L. KRALL that $P_{n}(x) \equiv(-1)^{n} y_{n}(x, 1,-1)$ where $y_{n}(x, a, b)$ are "generalized Bessel polynomials" (see [4]).

8. G. SzEGÖ, op. cit., p. 41-42.

9. Formula (14) holds for $n=2$ if we define $P_{0}(x) \equiv 1$.

10. G. SZEGÖ, op. cit., p. 341-342.

\section{On the Improvement of the Solutions to a Set of Simultaneous Linear Equations using the ILLIAC}

The basic method used for solving simultaneous linear equations on the University of Illinois' electronic digital computer, the ILLIAC, has already been described in detail by WHEELER and NASH [1]. The routine currently in use on the ILLIAC, programmed by Wheeler [2], makes use of the method of elimination to solve the set of $n$ simultaneous linear equations

$$
\sum_{j=0}^{n-1} a_{i j} x_{j}+a_{i n}=0 \quad i=0,1,2, \cdots, n-1
$$

in a manner very similar to that used by a human solving such a system.

In brief, the procedure used is as follows:

a) The augmented matrix

$$
\begin{aligned}
a_{i j} \quad i & =0,1,2, \cdots, n-1 \\
& j=0,1,2, \cdots, n
\end{aligned}
$$

